\title{
Development of a Freeze-Dried CRISPR-Cas12 Sensor for Detecting Wolbachia in the Secondary Science Classroom
}

\author{
Grant A. Rybnicky ${ }^{1,2,3}$, Radeen A. Dixon ${ }^{4}$, Robert M. Kuhn ${ }^{4,5}$, Ashty S. Karim ${ }^{1,2,6}$, \\ Michael C. Jewett ${ }^{1,2,6,7,8 *}$ \\ ${ }^{1}$ Chemistry of Life Processes Institute, Northwestern University, Evanston, IL, 60208, \\ USA \\ ${ }^{2}$ Center for Synthetic Biology, Northwestern University, Evanston, IL, 60208, USA \\ IInterdisciplinary Biological Sciences Graduate Program, Northwestern University, \\ Evanston, IL, 60208, USA \\ ${ }^{4}$ Centennial High School, 9310 Scott Rd, Roswell, GA 30076, United States \\ 5 Innovation Academy Fulton County Schools STEM Magnet High School, 125 Milton \\ Ave. Alpharetta, GA 30009, United States \\ ${ }^{6}$ Department of Chemical and Biological Engineering, Northwestern University, \\ Evanston, IL 60208, USA \\ ${ }^{7}$ Robert H. Lurie Comprehensive Cancer Center, Northwestern University, Chicago, IL \\ 60611, USA \\ ${ }^{8}$ Simpson Querrey Institute, Northwestern University, Chicago, IL 60611, USA \\ *Correspondence to: m-jewett@northwestern.edu, 847-467-5007
}




\begin{abstract}
Training the future synthetic biology workforce requires opportunity and exposure to biotechnology concepts and activities in secondary education. Detecting Wolbachia bacteria in arthropods using PCR has become a common way for secondary students to investigate and apply DNA technology in the science classroom. Despite this framework, cutting-edge biotechnologies like CRISPR-based diagnostics have yet to be widely implemented in the classroom. To address this gap, we present a freeze-dried CRISPR-Cas12 sensing reaction to complement traditional DNA technology education and teach synthetic biology concepts. The reactions accurately detect Wolbachia from arthropod-derived PCR samples in under 2 hours and can be stored at room temperature for over a month without appreciable degradation. The reactions are easy-to-use and cost less than $\$ 40$ to implement for a classroom of 22 students including the cost of reusable equipment. We see this technology as an accessible way to incorporate synthetic biology education into existing biology curriculum, which will expand biology educational opportunities in science, technology, engineering, and mathematics (STEM) education.
\end{abstract}

\title{
Keywords
}

CRISPR, Cas12, Nucleic Acid Detection, Secondary Science Education, Wolbachia 


\section{Introduction}

The global market for synthetic biology products is estimated to grow to over $\$ 30$ billion USD by 2026 from a current $\$ 9.5$ billion USD in $2021^{1}$. To support this growth, a workforce of graduating students must be inspired and trained to work in synthetic biology, and its associated science, technology, engineering, and mathematics (STEM) disciplines ${ }^{2-5}$. This requires student exposure to, and formative hands-on experiences with, STEM educational activities.

Many secondary schools in the United States have adopted STEM-related frameworks to teach science and engineering practices. For example, thousands of secondary science classrooms integrate DNA technology into curriculum through activities like the Wolbachia Project ${ }^{6}$. The Wolbachia Project teaches ecology, biotechnology, and bioinformatics concepts through surveying native arthropods for Wolbachia infection. Given the widespread range of these bacteria globally ${ }^{7}$, detection of Wolbachia in the secondary education classroom has become an accessible model for learning about DNA biotechnologies. The project includes collection and identification of arthropods from the students' local environment, bulk DNA extraction from the specimen, Polymerase Chain Reaction (PCR) amplification of a Wolbachia specific amplicon, and gel electrophoresis-based detection of the PCR amplicon (Figure 1A). By carrying out these activities, students learn about the existence of standard molecular biotechnology techniques as well as practice them in an engaging inquiry-based way. While curriculum like this teaches foundational techniques important to synthetic biology, translation of the most cutting-edge synthetic biology concepts has been limited.

A major barrier to teaching synthetic biology and other cutting edge STEM concepts is that education standards can be inflexible and make it difficult to interject new topics not explicit in the standard curriculum ${ }^{8}$. Despite this barrier, recent synthetic biology-based educational efforts like BioBits ${ }^{2-4}$, the BioBuilder Educational Foundation ${ }^{9}$, the International Genetically Engineered Machines competition, Amino Labs, and The ODIN are making progress. Yet, there are opportunities to do more. A particularly exciting area for expansion is in portable cell-free diagnostics $^{10,11}$. In recent years, point-of-use, cell-free diagnostics based on clustered regularly interspaced short palindromic repeats (CRISPR) CRISPR-associated (Cas) technologies have emerged to detect everything from human parasites ${ }^{12}$ to SARS-CoV-2 $2^{13,14}$. A key feature of CRISPR-based diagnostics is the ability to use Cas $12^{15}$ or Cas $13^{16}$ to convert DNA or RNA binding into an observable signal, respectively (Figure 1B). Incorporating synthetic biology innovations like this into existing biology lab activities could lead to improved classroom activities but more importantly the integration of synthetic biology education into the educational infrastructure that already exists.

Here, we present a cell-free, tube-based, and freeze-dried CRISPR-Cas12 (FD-CC12) DNA sensing reaction that can be directly integrated into a popular DNA technology educational activity developed by the Wolbachia Project to visualize the presence of Wolbachia DNA in arthropod-derived samples (Figure 1A). In contrast to gel electrophoresis-based visualization, FDCC12 reactions do not require specialized electrophoresis equipment, pipetting into small wells, DNA staining or band interpretation, and are ready to interpret in two or fewer hours. FD-CC12 reactions are less technically challenging to perform than gel electrophoresis, and a positive result is easily interpreted as fluorescence visible in the tube after incubation at $37^{\circ} \mathrm{C}$. This system modifies a traditional DNA technology educational activity and incorporates synthetic biology concepts through CRISPR-based diagnostics. In addition, FD-CC12 reactions are cheaper to implement than typical electrophoresis systems, easy to use, and are reliable in detecting Wolbachia infection within field-collected arthropod specimens. The reactions do not require refrigeration and are stable at room temperature for over a month. Using this FD-CC12 Wolbachia assay, secondary science students will gain hands-on experience with cutting edge synthetic biology and stable, low cost, reliable Wolbachia detection in arthropods. We anticipate that integrating synthetic biology into existing activities will be a powerful approach to teaching innovation in synthetic biology. 


\section{Results}

\section{Development of a Cas12 Diagnostic to Detect Wolbachia}

We set out to develop a CRISPR-Cas12 diagnostic for Wolbachia detection to implement in secondary classrooms. First, we developed a functional CRISPR-Cas12 diagnostic. Cas12, like other CRISPR nucleases, binds and cleaves DNA at a specific sequence encoded by a CRISPR RNA (crRNA) ${ }^{17}$. Once activated, Cas12 indiscriminately cleaves single stranded DNA (ssDNA) in solution (Figure 1B) ${ }^{15}$. This indiscriminate cleavage activity can be visualized within a diagnostic in a variety of ways, including the use of a ssDNA chemically modified with a fluorophore on one end and a fluorescence quencher on the other. In the uncleaved state, the ssDNA probe emits no fluorescence upon excitation as the quencher molecule is near the fluorophore and absorbs the fluorescent emission from the fluorophore. Once cleaved, the ssDNA probe is no longer intact, and the fluorescence quencher is not close enough to the fluorophore to absorb the fluorescent emission. To test for CRISPR-Cas 12 activity, we screened all possible crRNAs that can target the consensus 483 bp Wolbachia amplicon previously developed for the Wolbachia Project. Of the five locations with the appropriate TTTV Protospacer Adjacent Motif $(P A M)^{17}$ (Supplementary Figure S1), all possible crRNAs mediated detection of the Wolbachia amplicon (Figure 1C). Although all were functional, reaction endpoint fluorescence varied with different crRNA constructs. crRNA 286R yielded the highest endpoint fluorescence as well as fold activation. Thus, we selected crRNA 286R for further development.

\section{Characterization of Freeze-Dried Cas12 Diagnostics}

With a functional CRISPR-Cas12 diagnostic for Wolbachia detection in hand, we next wanted to assess the possibility of freeze-drying the system. Previous works have shown that cell-free systems, including CRISPR-based diagnostics, can be freeze-dried for increasing stability and portability ${ }^{18,19}$. Such features would be advantageous for preparation and delivery in the classroom setting. We freeze-dried CRISPR-Cas12 cell-free reactions in a VirTis BenchTop Pro lyophilizer at $\leq 100$ mtorr and $-80^{\circ} \mathrm{C}$ overnight or until fully freeze-dried. We then compared CRISPR-Cas 12 reactions prepared from fresh reagents and these pre-assembled, lyophilized reactions to determine whether the CRISPR-Cas 12 formulation is stable through the lyophilization process. Unfortunately, when lyophilized, the CRISPR-Cas12 formulation demonstrated no activity in the presence of activating DNA (Figure 2A). We hypothesized that the lack of activity was due to degradation of the Cas12 protein or the crRNA.

To address this bottleneck and improve the stability of the reactions, we evaluated diagnostic activity when reactions were lyophilized in the presence of different lyoprotectant formulations (Figure 2B). Specifically, we tested sucrose, trehalose, and dextran (70 kDa average weight) individually and in combination. We found that the activity of reactions supplemented with sucrose and trehalose individually increased with concentration, but dextran exhibited a concentration optimum of $25 \mathrm{mg} / \mathrm{mL}$ in the final reaction. Although combining $25 \mathrm{mg} / \mathrm{mL}$ each of sucrose and trehalose achieved similar endpoint fluorescence as $50 \mathrm{mg} / \mathrm{mL}$ of each respective lyoprotectant, combining $25 \mathrm{mg} / \mathrm{mL}$ of either sugar with $25 \mathrm{mg} / \mathrm{mL}$ dextran increased the retained activity. Combining $25 \mathrm{mg} / \mathrm{mL}$ each of sucrose and dextran exhibited approximately $75 \%$ recovery of the pre-lyophilization signal.

Additionally, all formulations containing dextran exhibited beneficial changes in freezedried pellet consistency. Rather than a powdery pellet subject to movement by static electricity, all lyophilized dextran formulations exhibited a bulky, solid pellet unaffected by static charges. This made manipulation of pellets much easier and decreased the risk of static electricity ejecting portions of the pellet from the tube. In making the pellet easier to work with, the inclusion of dextran in the lyoprotectant formulation made the FD-CC12 reactions more amenable to use in 
secondary science classrooms. Moving forward we chose to use $25 \mathrm{mg} / \mathrm{mL}$ each of sucrose and dextran to formulate our freeze-dried reactions.

We next characterized the final formulation on freeze-dried CRISPR-Cas12 (FD-CC12) reaction stability over time. After lyophilization we stored reactions at either 20 or $37^{\circ} \mathrm{C}$ in vacuum sealed bags with a desiccant card for two months, monitoring activity over time. When stored at room temperature, the lyophilized reactions retained approximately $75 \%$ activity in the on state after 2 months of storage without appreciable leak in off state (Figure $2 \mathrm{C}$ ). When stored at $37^{\circ} \mathrm{C}$ for the same amount of time, the reactions retained over $40 \%$ activity in the on state without leak.

\section{Assessment of Freeze-Dried Cas12 Diagnostics with different PCR mixes}

In the Wolbachia Project, students amplify DNA using PCR following DNA extraction from arthropods. Different classrooms may use different PCR reaction mixtures, based on price and preference. To assess generalizability of our FD-CC12 reactions and facilitate integration into existing classroom activities, we therefore tested compatibility with common PCR reaction mixtures. We evaluated whether several commonly used PCR kits (Q5 Hotstart, NEB Taq, MiniPCR EZ PCR, Thermo DreamTaq, and Promega GoTaq) could be used to rehydrate the FDCC12 reactions and maintain functionality. Final endpoint fluorescence varied among FD-CC12 reactions rehydrated with different PCR kits, all achieving at least $60 \%$ of the signal achieved by reactions rehydrated with purified DNA (Figure 2D). Despite this variability in endpoint fluorescence, all the reactions produced observable signal in the on state and negligible signal in the off state. These data highlight the robustness of our FD-CC12 diagnostic for detecting Wolbachia.

\section{Assessment of Freeze-Dried Cas12 Diagnostics in the Classroom}

With stable and robust FD-CC12 reactions established, we next wanted to test compatibility with the Wolbachia Project educational framework. We first wanted to make sure that Wolbachia DNA from arthropod-derived samples could be detected. When rehydrated with a PCR reaction that used bulk DNA from a Wolbachia infected Nasonia vitripennis wasp as template, the FD-CC12 reaction achieved endpoint yields comparable to those observed in the PCR kit screen (Figure 3A). Using a Wolbachia-free $N$. vitripennis wasp-derived PCR, the FDCC12 reaction showed signal above background observable by fluorescent plate reader but not by eye.

Once validated with control insects, we next assessed the ability of the workflow to be completed in a high school classroom setting by a high school student. FD-CC12 reactions were prepared at Northwestern University and shipped to Centennial High School (CHS) in Roswell, Georgia. At CHS, a student collected and identified 22 arthropods across 6 orders (Supplementary Table S2), extracted bulk DNA from each specimen, and performed PCR to amplify the Wolbachia amplicon as is standard practice in the Wolbachia Project workflow. Those PCR products were then used to rehydrate FD-CC12 reactions and were also visualized using gel electrophoresis. Out of the 22 samples tested, 21 agreed between FD-CC12 reaction and gel electrophoresis (Figure 3B). For sample ID\# 11, the gel indicated Wolbachia infection while the FD-CC12 reaction indicated no infection. Upon sequencing the Wolbachia amplicon for sample ID\# 11, it was revealed that there were 5 mutations within the protospacer region the crRNA 286R targeted (Supplementary Figure S2). As Wolbachia are a diverse genus of bacteria, it is not unreasonable to expect that some isolates would evade detection by any single crRNA. Using multiple crRNAs in the same reaction to create OR gate logic, it may be possible to detect a wider diversity of isolates. Given the compatibility of the FD-CC12 reactions with arthropod-derived samples, successful completion by a high school student, and high accuracy of sensing reactions, this demonstrates that FD-CC12 reactions can be integrated into the Wolbachia Project laboratory activities. 


\section{Discussion}

We developed a FD-CC12 Wolbachia DNA sensing reaction that can be used to teach synthetic biology concepts in the secondary science classroom. As designed, the sensing reactions detect a PCR amplicon commonly used to diagnose Wolbachia infection in fieldcollected arthropods as a demonstration of DNA technologies (Figure 1A). We identified and evaluated 5 functional crRNAs that can be used to detect this amplicon with Cas12 (Figure 1C). Additionally, we identified a lyoprotectant additive formulation that both recovers about $75 \%$ of reaction endpoint fluorescence after lyophilization (Figure 2A\&B) and is stable at room temperature for over a month without appreciable degradation (Figure 2C). Using this formulation, we evaluated the reaction's compatibility with commonly used PCR kits, finding that the freezedried reactions could be rehydrated with virtually any unpurified PCR product and still distinguish the presence of the Wolbachia amplicon in the sample (Figure 2D). To validate the reaction can be applied to the established classroom activity, we rehydrated freeze-dried reactions with arthropod-derived samples. Nasonia wasps with known Wolbachia infection states yielded results as expected (Figure 3A). Screening of 22 field-collected arthropods across 6 orders for Wolbachia infection by a high school student using gel electrophoresis and FD-CC12 reactions yielded a 95\% accuracy rate (Figure 3B).

While we recommend that FD-CC12 sensing reactions be used to complement gel electrophoresis in DNA technology education, we do recognize that specific features of our sensing reactions may make them a more attractive readout than gel electrophoresis to secondary science educators. The FD-CC12 reactions primarily lower two barriers DNA visualization in the classroom: 1) FD-CC12 reactions are less technically difficult to set up and run than gel electrophoresis and 2) FD-CC12 reactions are less expensive. While commonplace in the molecular biology research lab, gel electrophoresis poses a few technical hurdles to implementation that science educators may face. Foremost, gel electrophoresis is intimidating to educators who have not had hands-on training in a laboratory or elsewhere. The ability to properly prepare and cast an agarose gel, pipette samples into the well of a gel, and consistently stain and visualize gels takes hands-on practice before it becomes routine. Collectively, these technical hurdles may cause teachers to seek more expensive alternatives (precast gels for example) or to avoid implementation of gel electrophoresis altogether. Alternatively, the FD-CC12 reactions contain all necessary components within a single tube, are easier to pipette analyte into, and are visualized by handheld blue light imager without the addition of stains. The use of freeze-dried cell-free reactions has the potential to increase teacher confidence in their laboratory skills and encourage them to attempt laboratory activities they may have been hesitant to try before like gel electrophoresis. Similarly, the initial monetary investment required to implement FD-CC12 sensing in the classroom is lower than implementing gel electrophoresis. Requiring only incubation at $37^{\circ} \mathrm{C}$ and a blue light imager like the MiniPCR P51 ${ }^{\mathrm{TM}}$ Molecular Fluorescence Viewer, the FD-CC12 reactions have a startup cost of $\sim \$ 38$ for a 22-student class. Alternatively, the equipment to cast gels, generate current, house the gel in buffer, and visualize the gel as well as initial reagents cost $\sim \$ 1000$ or $\sim \$ 300$ for the same sized class for traditional or education optimized gel electrophoresis equipment, respectively (Table 1). Assuming one sample per student and class size of 22 students, the CRISPR-Cas12-based activity is cheaper to run for 848 students or fewer than education optimized gel electrophoresis set up. The price of FD-CC12 reactions can be further reduced about $10 \mathrm{X}$ by applying state-of-the-art cell-free technology like the use of paper instead of tubes ${ }^{20}$. That being said, gel electrophoresis systems are generalizable to visualizing almost any DNA sample while the FD-CC12 sensing reaction presented is specific to Wolbachia amplicon detection given the crRNA used.

Although we focused on detection of Wolbachia in the secondary science classroom, we note that the technology described has potential applications beyond the scope presented. Within science education, the DNA that is detected can easily be changed by altering the crRNA in the reaction. There are numerous well established laboratory activities that use gel electrophoresis 
to detect double stranded DNA PCR product. Given the presence of a functional PAM sequence in the DNA analyte and appropriate design of a crRNA, the technology described here can be extended to other educational activities beyond Wolbachia detection. The technology is not limited to the classroom either. As the freeze-dried cell-free reactions are still functional after 56 days storage at $37^{\circ} \mathrm{C}$ (Figure $2 \mathrm{C}$ ), it is feasible to use these reactions in on-site biological field research. In addition, FD-CC12 reactions could be implemented in field stations where gel electrophoresis may be difficult or impractical. As mentioned before, other DNAs can alternatively be detected by exchanging the crRNA in the system. This can lead to applications such as tracking of specific organisms in the environment by DNA detection, identification of visibly indistinguishable species, or monitoring the spread of mobile genetic elements in the environment.

Taken together, FD-CC12 reactions have the potential to be a simple, cost-effective companion to gel electrophoresis that provide a hands-on way to teach CRISPR-based nucleic acid detection and concepts of synthetic biology. In integrating this activity with the established DNA technology workflow designed by the Wolbachia Project, we anticipate widespread adoption of CRISPR-Cas12-based detection into the classroom, further strengthening the effort to expose school age children to synthetic biology concepts and priming the future STEM workforce. 


\section{Methods}

\section{Cas12 Sensing Reactions}

Fresh Cas 12 reactions were assembled by mixing reaction buffer $(40 \mathrm{mM}$ Tris- $\mathrm{HCl}, 60$ $\mathrm{mM} \mathrm{NaCl}, 6 \mathrm{mM} \mathrm{MgCl}$, pH 7.3), 45 nM AsCas12a V3 (IDT), 45 nM crRNA (IDT), $1 \mu \mathrm{M}$ ssDNA Fluorophore-Quencher (FQ) Probe (IDT), and lyoprotectant. Prior to addition of FQ Probe and lyoprotectant, the mixture was equilibrated at $37^{\circ} \mathrm{C}$ for 15 minutes. Upon addition of the analyte, the reaction was incubated at $37^{\circ} \mathrm{C}$ and fluorescence was measured (490 nm Excitation, $525 \mathrm{~nm}$ Emission) using a Synergy H1 microplate reader (BioTek, USA) and Gen5 v. 2.09 (BioTek) software.

Lyophilized Cas 12 reactions were assembled as described above and then flash frozen with liquid nitrogen in PCR strip tubes (Thermo Scientific, AB2000) with a small hole melted in the cap of each tube. The reactions were lyophilized using a VirTis BenchTop Pro lyophilizer (SP Scientific) at $\leq 100 \mathrm{mtorr}$ and $-80{ }^{\circ} \mathrm{C}$ overnight or until fully freeze-dried. Following lyophilization, tubes were packaged in Food Saver $®$ bags with a desiccant card, vacuum sealed and stored at the indicated temperature. When ready for use, tubes were removed from the Food Saver ${ }^{\circ}$ bags and rehydrated with the same volume of analyte as the mixture prior to lyophilization. Reactions were incubated at $37^{\circ} \mathrm{C}$ and fluorescence was measured as described above.

\section{Arthropod Collection and Wolbachia DNA Amplification}

Arthropods belonging to six different orders (Supplemental Table S2) were collected from Roswell, GA and Brevard, NC. Each specimen was preserved in $75 \% \mathrm{EtOH}$ in a $1.5 \mathrm{~mL}$ collection tube and frozen at $-20{ }^{\circ} \mathrm{C}$. Samples were visually identified to taxonomic order and later identified further by $\mathrm{COI}$ barcoding. PCR using the primers described by Folmer ${ }^{21}$ was performed and the product was sanger sequenced (GeneWiz). Consensus sequences for each specimen were obtained using DNA Subway (CyVerse/DNA learning Center) and NIH nucleotide BLAST ${ }^{22}$ was used to taxonomically identify the arthropod. Wolbachia-infected and Wolbachia-free Nasonia vitripennis wasps were obtained from the Wolbachia Project through the Bordenstein Lab at Vanderbilt University. Bulk DNA was extracted from each specimen by manual grinding using sterile, plastic minipestles for 2 minutes in a $1.5 \mathrm{~mL}$ microcentrifuge tube containing $200 \mu \mathrm{L}$ of lysis buffer/EDTA mix and subsequent purification with the Monarch Genomic DNA Purification Kit (catalog \#T3010) as directed. A $100 \mu \mathrm{L}$ DNA elution was collected stored at $-20{ }^{\circ} \mathrm{C}$ until used for polymerase chain reaction (PCR).

PCR was performed using $2 \mu \mathrm{L}$ of eluted DNA, Wspec-F (5'-CAT ACC TAT TCG AAG GGA TAG-3') and Wspec-R (5'-AGC TTC GAG TGA AAC CAA TTC-3') primers and MiniPCR EZ PCR Master Mix (catalog RG-1000-01). MiniPCR Mini8 thermocyclers were used for PCR with the following protocol: initial denaturation at $94{ }^{\circ} \mathrm{C}$ for $120 \mathrm{sec}$ and 34 cycles of denaturation 95 ${ }^{\circ} \mathrm{C}$ for $30 \mathrm{sec}$, annealing $55^{\circ} \mathrm{C}$ for $45 \mathrm{sec}$, extension $72{ }^{\circ} \mathrm{C}$ for $60 \mathrm{sec}$ and final extension $72{ }^{\circ} \mathrm{C}$ for $600 \mathrm{sec}$. PCR product was frozen at $-20^{\circ} \mathrm{C}$ for further use.

\section{Gel Electrophoresis and Cas12 Detection of Wolbachia DNA in the Classroom}

Gel electrophoresis assays were completed in a secondary science education classroom at Centennial High School in Roswell, GA using the MiniPCR blueGel ${ }^{\mathrm{TM}}$ electrophoresis system with built-in transilluminator. Gels were poured using GelGreen ${ }^{\circledR}$ Agarose Tabs ${ }^{\mathrm{TM}}$ to a concentration of $2 \%$ agarose. $5 \mu \mathrm{L}$ of each PCR product was loaded into a well and were electrophoresed for $30 \mathrm{~min}$. The presence of Wolbachia in the arthropod specimen was determined by presence of a $438 \mathrm{bp}$ amplicon using the built in blue light transilluminator.

Freeze-Dried Cas 12 reactions were assembled and packaged at Northwestern University in Evanston, IL. The reactions were then shipped at room temperature to Centennial High School in Roswell, GA. Reactions were stored at room temperature for 9 days prior to rehydration. PCR strip tubes containing a freeze-dried pellet of the CRISPR-Cas12 system were rehydrated using $10 \mu \mathrm{L}$ of PCR product. Tubes were spun briefly at $10,000 \mathrm{rpm}$ and moved to a heat block to 
bioRxiv preprint doi: https://doi.org/10.1101/2021.10.06.463384; this version posted October 9, 2021. The copyright holder for this preprint

(which was not certified by peer review) is the author/funder, who has granted bioRxiv a license to display the preprint in perpetuity. It is made available under aCC-BY-NC-ND 4.0 International license.

incubate at $37{ }^{\circ} \mathrm{C}$ for 2 hours. Fluorescence was detected using a MiniPCR p51 fluorescence viewer and photographs taken using a blue light transilluminator. 


\author{
Abbreviations \\ PCR - Polymerase Chain Reaction \\ CRISPR - Clustered Regularly Interspaced Short Palindromic Repeats \\ Cas - CRISPR Associated Protein \\ FD-CC12 - Freeze-Dried CRISPR-Cas12 \\ STEM - Science Technology Engineering and Mathematics \\ K-12 - Kindergarten through Twelfth Grade \\ ssDNA - Single Stranded DNA \\ FQ - Fluorophore Quencher \\ PAM - Protospacer Adjacent Motif \\ crRNA - CRISPR RNA
}

\title{
Author Information
}

Grant A. Rybnicky - Interdisciplinary Biological Sciences Graduate Program, Northwestern University, 2205 Tech Drive, Hogan Hall 2100, Evanston, Illinois 60208, United States;

Chemistry of Life Processes Institute, Northwestern University, 2170 Campus Drive, Evanston, Illinois 60208-3120, United States; Center for Synthetic Biology, Northwestern University, 2145 Sheridan Road, Technological Institute E136, Evanston, Illinois 60208-3120, United States

Radeen A. Dixon - Student, Centennial High School, 9310 Scott Rd, Roswell, GA 30076, United States

Robert M. Kuhn - Teacher, Centennial High School, 9310 Scott Rd, Roswell, GA 30076, United States; Teacher, Innovation Academy Fulton County Schools STEM Magnet High School, 125 Milton Ave. Alpharetta, GA 30009, United States

Ashty S. Karim - Department of Chemical and Biological Engineering, Northwestern University, 2145 Sheridan Road, Technological Institute E136, Evanston, Illinois 60208-3120, United States; Chemistry of Life Processes Institute, Northwestern University, 2170 Campus Drive, Evanston, Illinois 60208-3120, United States; Center for Synthetic Biology, Northwestern University, 2145 Sheridan Road, Technological Institute E136, Evanston, Illinois 60208-3120, United States

Michael C. Jewett - Department of Chemical and Biological Engineering, Northwestern University, 2145 Sheridan Road, Technological Institute E136, Evanston, Illinois 60208-3120, United States; Chemistry of Life Processes Institute, Northwestern University, 2170 Campus Drive, Evanston, Illinois 60208-3120, United States; Center for Synthetic Biology, Northwestern University, 2145 Sheridan Road, Technological Institute E136, Evanston, Illinois 60208-3120, United States; Member, Robert H. Lurie Comprehensive Cancer Center, Northwestern University, 676 N. St. Clair Street, Suite 1200, Chicago, Illinois 60611-3068, United States; Simpson Querrey Institute, Northwestern University, 303 E. Superior Street, Suite 11-131, Chicago, Illinois 60611-2875, United States

\section{Author Contribution}

GAR, RAD, RMK, and MCJ conceived the work presented. GAR, RAD, and RMK ran experiments. GAR, RAD, RMK, ASK and MCJ wrote the paper.

\section{Competing Interests}

M.C.J. is a cofounder of SwiftScale Biologics, Stemloop, Inc., Design Pharmaceuticals, and Pearl Bio. M.C.J.'s interests are reviewed and managed by Northwestern University in accordance with their conflict of interest policies. 


\section{Acknowledgement}

We would like to acknowledge MiniPCR for their contribution of materials and reagents. We would also like to thank Sarah Bordenstein and the Wolbachia Project for helpful conversation and for $N$. vitripennis specimens. We would also like to acknowledge the Centennial High School DNA Club for providing collaborative biology research opportunities for high school students. The authors would like to acknowledge members of the Jewett Lab for helpful discussions. M.C.J. acknowledges support from the Department of Energy Grants DE-SC0018249, DE-NA0003525, and 8J-30009-0029A. the DOE Joint Genome Institute ETOP program, the Office of Energy Efficiency and Renewable Energy Grant DE-EE0008343, the David and Lucile Packard Foundation, the Camille Dreyfus Teacher-Scholar Program, the Defense Threat Reduction Agency Grants HDTRA1-15-10052/P00001 and HDTRA-12-01-0004, the Army Research Office Grants W911NF-20-1-0195, W911NF-18-1-0200, and W911NF-16-1-0372, the National Science Foundation Grants 1936789 and 1844336, and the Air Force Office for Research Grant FA238621-1-4078, Army Contracting Command Contract W52P1J-21-9-3023. The work conducted by the U.S. Department of Energy Joint Genome Institute, a DOE Office of Science User Facility, is supported by the Office of Science of the U.S. Department of Energy under Contract No. DEAC02-05CH11231. G.A.R was supported by the National Science Foundation Graduate Research Fellowship Program under Grant No. DGE-1842165. 


\section{References}

(1) Synthetic Biology Market by Tools (Oligonucleotides, Enzymes, Synthetic Cells), Technology (Gene Synthesis, Genome Engineering), Application(Tissue Regeneration, Biofuel, Consumer Care, Food \& Agriculture, Environmental) and Region - Global Forecast to 20; 2021.

(2) $\quad$ C., S. J.; Ally, H.; Q., N. P.; S., D. R.; J., H. K.; C., F. T.; Mary, A.; Ada, K.; Quinn, M.; S., P. J.; Palak, P.; Richa, P.; Deema, Q.; Tyler, Z.; Julie, B.; Thomas, M.; Ashlee, M.-B.; Aparna, P.; Kara, R.; Miriam, S.; Lance, B.; R., H. L.; F., C. J.; Nuhie, F.; Suzanne, F.; Elissa, G.; Marie, G. E.; Teresa, G.; Jenna, K.; Binh, N.; Sujud, O.; Christina, P.; Angela, P.; Sheila, S.; Adriane, S.; TaRhonda, W.; Nina, D.; Keith, P.; J., C. J.; C., J. M. BioBits ${ }^{\mathrm{TM}}$ Bright: A Fluorescent Synthetic Biology Education Kit. Sci. Adv. 2021, 4 (8), eaat5107. https://doi.org/10.1126/sciadv.aat5107.

(3) Ally, H.; Q., N. P.; C., S. J.; K., T. M.; Nina, D.; Tom, F.; J., D. A.; J., H. K.; S., D. R.; Keith, P.; C., J. M.; J., C. J. BioBits ${ }^{\mathrm{TM}}$ Explorer: A Modular Synthetic Biology Education Kit. Sci. Adv. 2021, 4 (8), eaat5105. https://doi.org/10.1126/sciadv.aat5105.

(4) Stark, J. C.; Huang, A.; Hsu, K. J.; Dubner, R. S.; Forbrook, J.; Marshalla, S.; Rodriguez, F.; Washington, M.; Rybnicky, G. A.; Nguyen, P. Q.; Hasselbacher, B.; Jabri, R.; Kamran, R.; Koralewski, V.; Wightkin, W.; Martinez, T.; Jewett, M. C. BioBits Health: Classroom Activities Exploring Engineering, Biology, and Human Health with Fluorescent Readouts. ACS Synth. Biol. 2019, 8 (5), 1001-1009. https://doi.org/10.1021/acssynbio.8b00381.

(5) Collias, D.; Marshall, R.; Collins, S. P.; Beisel, C. L.; Noireaux, V. An Educational Module to Explore CRISPR Technologies with a Cell-Free Transcription-Translation System. Synth. Biol. 2019, 4 (1). https://doi.org/10.1093/synbio/ysz005.

(6) Lemon, A.; Bordenstein, S. R.; Bordenstein, S. R. Discover the Microbes Within! The Wolbachia Project: Citizen Science and Student-Based Discoveries for 15 Years and Counting. Genetics 2020, 216 (2), 263 LP - 268. https://doi.org/10.1534/genetics.120.303649.

(7) Kaur, R.; Shropshire, J. D.; Cross, K. L.; Leigh, B.; Mansueto, A. J.; Stewart, V.; Bordenstein, S. R.; Bordenstein, S. R. Living in the Endosymbiotic World of Wolbachia: A Centennial Review. Cell Host Microbe 2021, 29 (6), 879-893. https://doi.org/https://doi.org/10.1016/j.chom.2021.03.006.

(8) Margot, K. C.; Kettler, T. Teachers' Perception of STEM Integration and Education: A Systematic Literature Review. Int. J. STEM Educ. 2019, 6 (1), 2. https://doi.org/10.1186/s40594-018-0151-2.

(9) Kuldell, N.; Bernstein, R.; Ingram, K.; Hart, K. M. BioBuilder, 1st ed.; Loukides, M., MacDonald, B., Eds.; O'Reilly Media, Inc.: Sebastopol, 2015.

(10) Silverman, A. D.; Karim, A. S.; Jewett, M. C. Cell-Free Gene Expression: An Expanded Repertoire of Applications. Nat. Rev. Genet. 2020, 21 (3), 151-170. https://doi.org/10.1038/s41576-019-0186-3.

(11) Kaminski, M. M.; Abudayyeh, O. O.; Gootenberg, J. S.; Zhang, F.; Collins, J. J. CRISPRBased Diagnostics. Nat. Biomed. Eng. 2021, 5 (7), 643-656.

https://doi.org/10.1038/s41551-021-00760-7.

(12) Lee, R. A.; Puig, H. De; Nguyen, P. Q.; Angenent-Mari, N. M.; Donghia, N. M.; McGee, J. P.; Dvorin, J. D.; Klapperich, C. M.; Pollock, N. R.; Collins, J. J. Ultrasensitive CRISPRBased Diagnostic for Field-Applicable Detection of \&lt;Em\&gt;Plasmodium\&lt;/Em\&gt; Species in Symptomatic and Asymptomatic Malaria. Proc. Natl. Acad. Sci. 2020, 117 (41), 25722 LP - 25731. https://doi.org/10.1073/pnas.2010196117.

(13) Ackerman, C. M.; Myhrvold, C.; Thakku, S. G.; Freije, C. A.; Metsky, H. C.; Yang, D. K.; Ye, S. H.; Boehm, C. K.; Kosoko-Thoroddsen, T.-S. F.; Kehe, J.; Nguyen, T. G.; Carter, A.; Kulesa, A.; Barnes, J. R.; Dugan, V. G.; Hung, D. T.; Blainey, P. C.; Sabeti, P. C. Massively Multiplexed Nucleic Acid Detection with Cas13. Nature 2020, 582 (7811), 277- 
282. https://doi.org/10.1038/s41586-020-2279-8.

(14) Broughton, J. P.; Deng, X.; Yu, G.; Fasching, C. L.; Servellita, V.; Singh, J.; Miao, X.; Streithorst, J. A.; Granados, A.; Sotomayor-Gonzalez, A.; Zorn, K.; Gopez, A.; Hsu, E.; Gu, W.; Miller, S.; Pan, C.-Y.; Guevara, H.; Wadford, D. A.; Chen, J. S.; Chiu, C. Y. CRISPR-Cas12-Based Detection of SARS-CoV-2. Nat. Biotechnol. 2020, 38 (7), 870874. https://doi.org/10.1038/s41587-020-0513-4.

(15) Chen, J. S.; Ma, E.; Harrington, L. B.; Da Costa, M.; Tian, X.; Palefsky, J. M.; Doudna, J. A. CRISPR-Cas12a Target Binding Unleashes Indiscriminate Single-Stranded DNase Activity. Science 2018, 360 (6387), 436-439. https://doi.org/10.1126/science.aar6245.

(16) O., A. O.; S., G. J.; Silvana, K.; Julia, J.; M., S. I.; T., C. D. B.; Sergey, S.; S., M. K.; Ekaterina, S.; Leonid, M.; Konstantin, S.; Aviv, R.; S., L. E.; V., K. E.; Feng, Z. C2c2 Is a Single-Component Programmable RNA-Guided RNA-Targeting CRISPR Effector. Science (80-. ). 2016, 353 (6299), aaf5573. https://doi.org/10.1126/science.aaf5573.

(17) Zetsche, B.; Gootenberg, J. S.; Abudayyeh, O. O.; Slaymaker, I. M.; Makarova, K. S.; Essletzbichler, P.; Volz, S. E.; Joung, J.; van der Oost, J.; Regev, A.; Koonin, E. V; Zhang, F. Cpf1 Is a Single RNA-Guided Endonuclease of a Class 2 CRISPR-Cas System. Cell 2015, 163 (3), 759-771. https://doi.org/10.1016/j.cell.2015.09.038.

(18) C., S. J.; Thapakorn, J.; D., M. T.; M., H. J.; F., W. K.; S., M. B.; M., M. A.; S., D. R.; J., H. K.; C., S. T.; D., J. B.; P., D. M.; C., J. M. On-Demand Biomanufacturing of Protective Conjugate Vaccines. Sci. Adv. 2021, 7 (6), eabe9444. https://doi.org/10.1126/sciadv.abe9444.

(19) Gootenberg, J. S.; Abudayyeh, O. O.; Lee, J. W.; Essletzbichler, P.; Dy, A. J.; Joung, J.; Verdine, V.; Donghia, N.; Daringer, N. M.; Freije, C. A.; Myhrvold, C.; Bhattacharyya, R. P.; Livny, J.; Regev, A.; Koonin, E. V; Hung, D. T.; Sabeti, P. C.; Collins, J. J.; Zhang, F. Nucleic Acid Detection with CRISPR-Cas13a/C2c2. Science 2017, 356 (6336), 438-442. https://doi.org/10.1126/science.aam9321.

(20) Pardee, K.; Green, A. A.; Ferrante, T.; Cameron, D. E.; DaleyKeyser, A.; Yin, P.; Collins, J. J. Paper-Based Synthetic Gene Networks. Cell 2014, 159 (4), 940-954. https://doi.org/10.1016/j.cell.2014.10.004.

(21) Folmer, O.; Black, M.; Hoeh, W.; Lutz, R.; Vrijenhoek, R. DNA Primers for Amplification of Mitochondrial Cytochrome c Oxidase Subunit I from Diverse Metazoan Invertebrates. Mol. Mar. Biol. Biotechnol. 1994, 3 (5), 294-299.

(22) Altschul, S. F.; Gish, W.; Miller, W.; Myers, E. W.; Lipman, D. J. Basic Local Alignment Search Tool. J. Mol. Biol. 1990, 215 (3), 403-410. https://doi.org/10.1016/S00222836(05)80360-2. 


\section{Figures and Tables}

A)

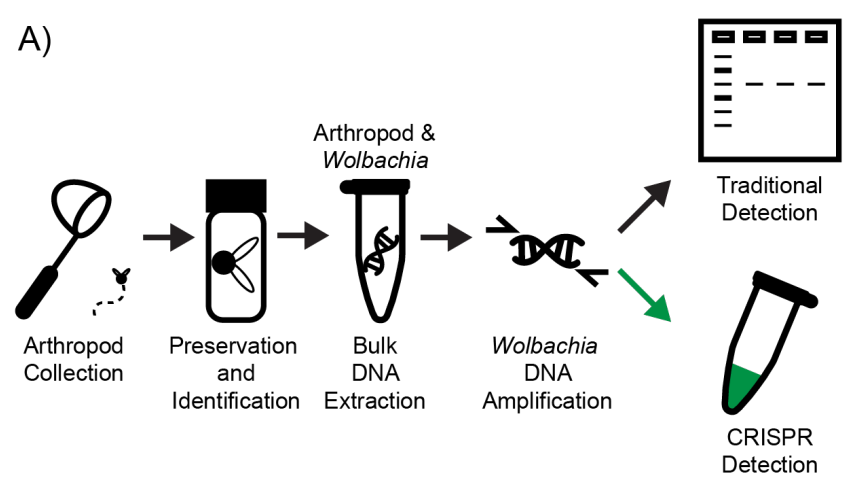

B)
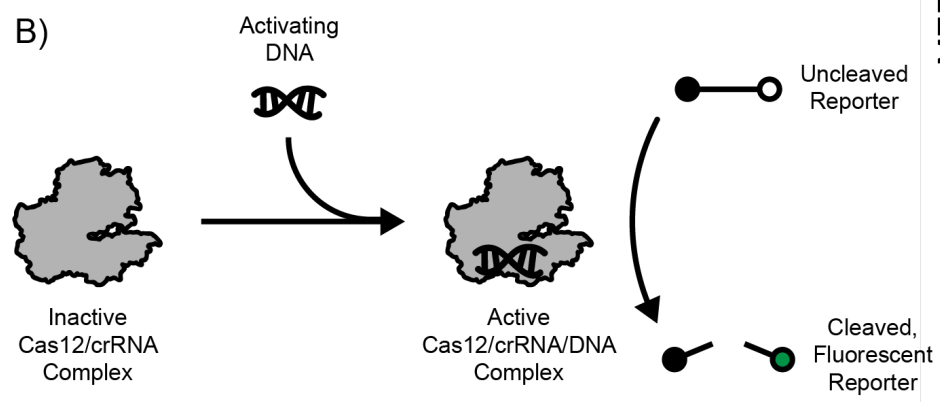

C)

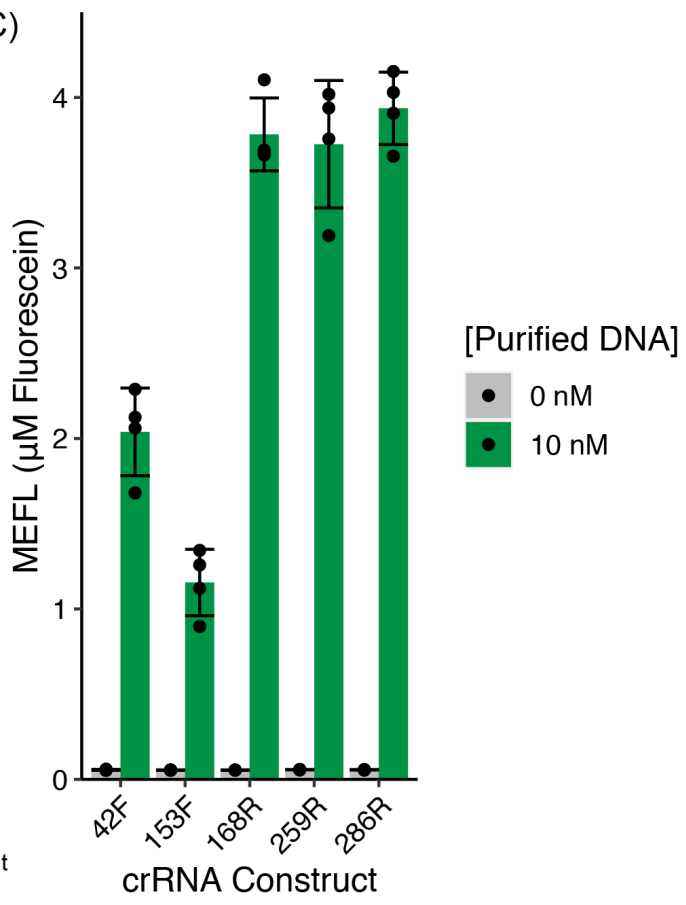

Figure 1. CRISPR-Cas12 diagnostics can be integrated into existing high school biology curriculum. A) Cas12-based DNA sensing reactions can theoretically be used in addition to gel electrophoresis to detect Wolbachia infection in student collected arthropod samples. B) Cas 12 is activated by the presence of DNA complementary to the crRNA and indiscriminately cleaves ssDNA. A ssDNA quencher-fluorophore probe can convert this activity to observable fluorescence. C) All possible crRNAs against the Wolbachia amplicon were tested for activity. Endpoint fluorescence after incubation at $37{ }^{\circ} \mathrm{C}$ for 2 hours is reported. Error bars represent standard deviation of 4 replicates. Purified synthetic DNA with the same sequence as the consensus Wolbachia amplicon was used as an activator. 

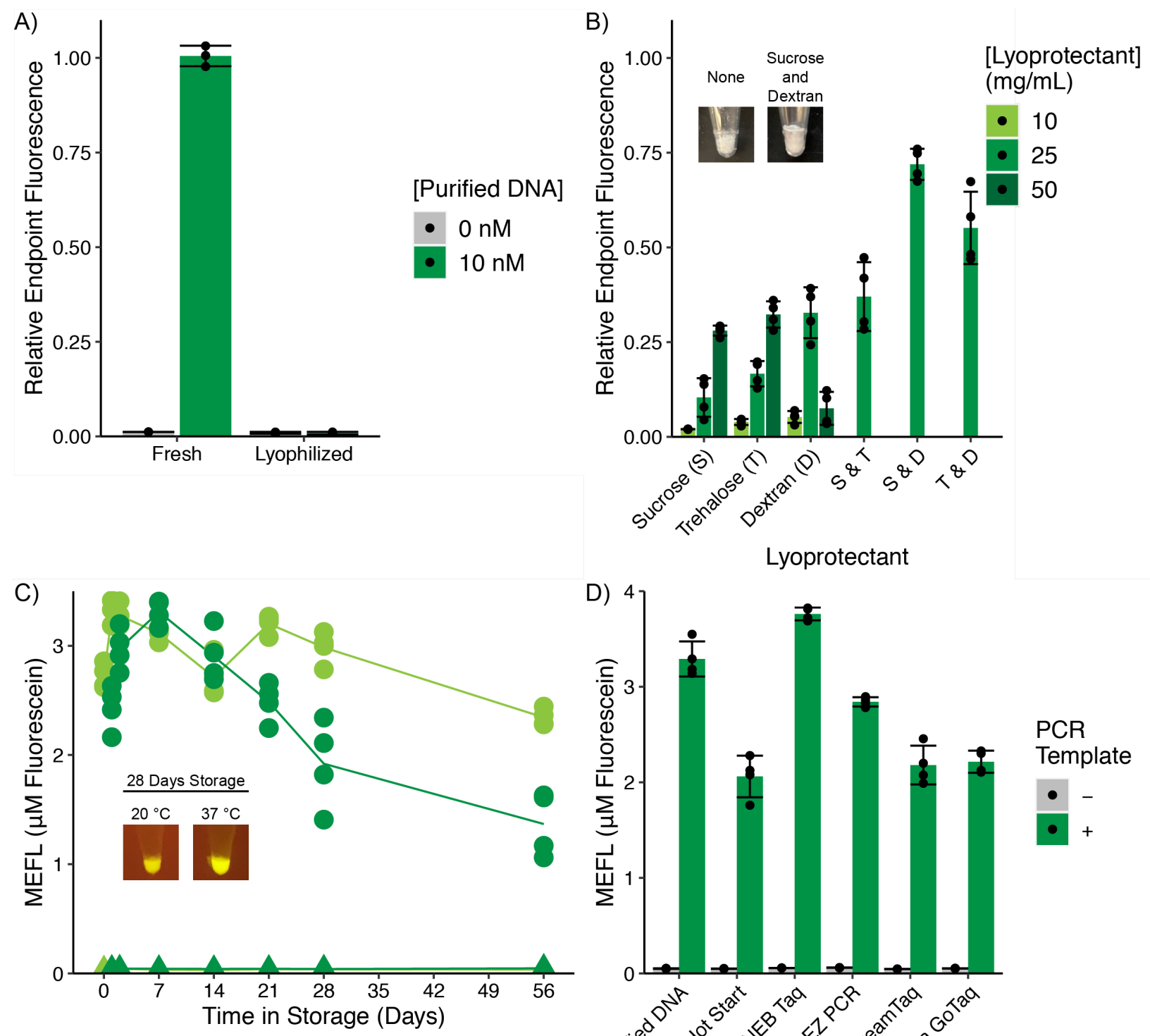

Storage Temperature $\left({ }^{\circ} \mathrm{C}\right) \quad$ [Purified DNA]
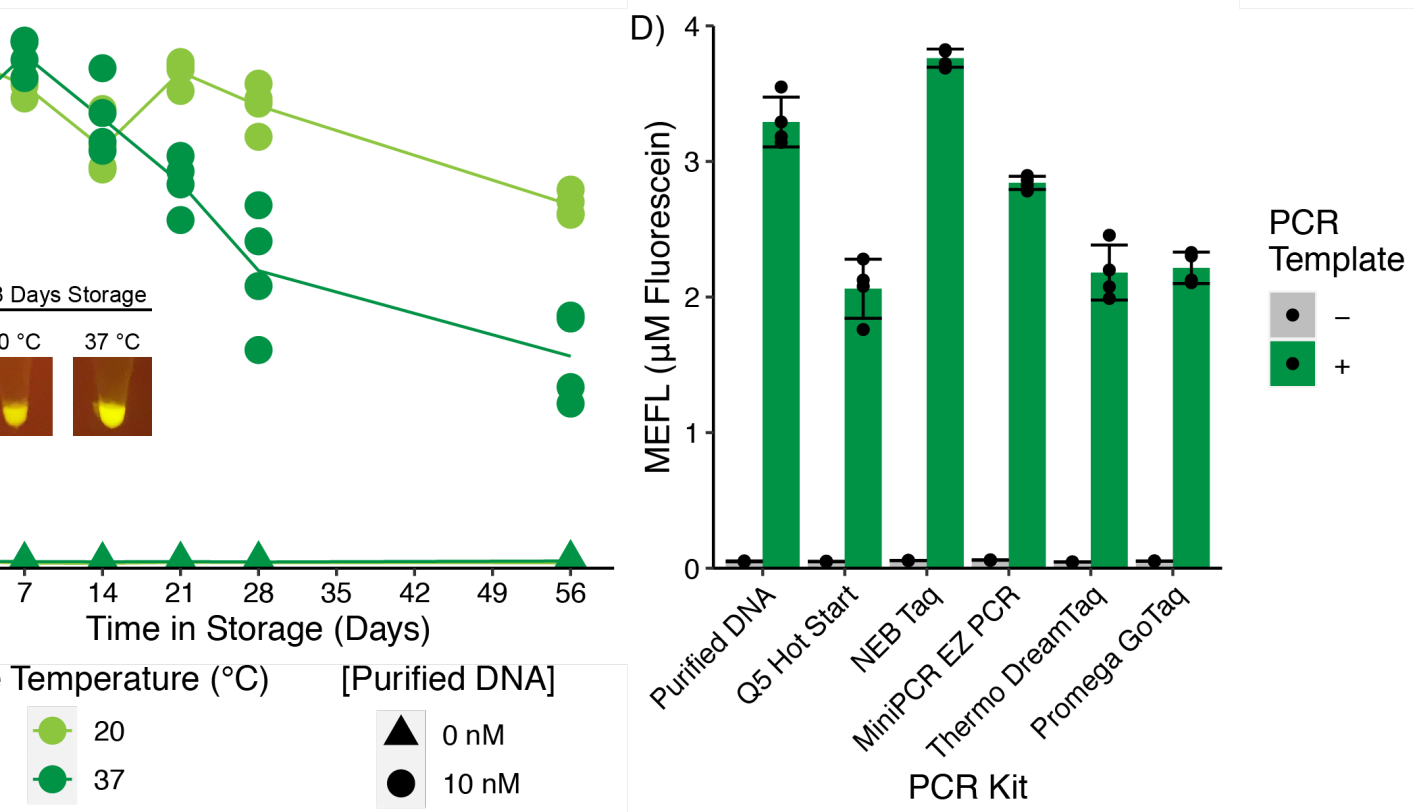

Figure 2. Development and characterization of freeze-dried CRISPR-Cas12 sensing reactions. Endpoint fluorescence was determined after incubation at $37^{\circ} \mathrm{C}$ for 2 hours. Error bars represent standard deviation of 4 replicates. B) Inset photos are representative images of FDCC12 reaction prior to rehydration. C) Lyophilized reactions supplemented with $25 \mathrm{mg} / \mathrm{mL}$ each of sucrose and dextran were stored in PCR tubes vacuum sealed in Food Saver® bags with a desiccant card. All data from 4 replicates of each reaction are plotted. The line connects the average value at each time point. Inset photos are representative images of reactions rehydrated with purified DNA and incubated at $37^{\circ} \mathrm{C}$ for 2 hours viewed with a portable blue light imager. D) Lyophilized reactions supplemented with $25 \mathrm{mg} / \mathrm{mL}$ each of sucrose and dextran were rehydrated with the same volume of purified DNA or unpurified PCR product as the initial reaction volume prior to lyophilization. 


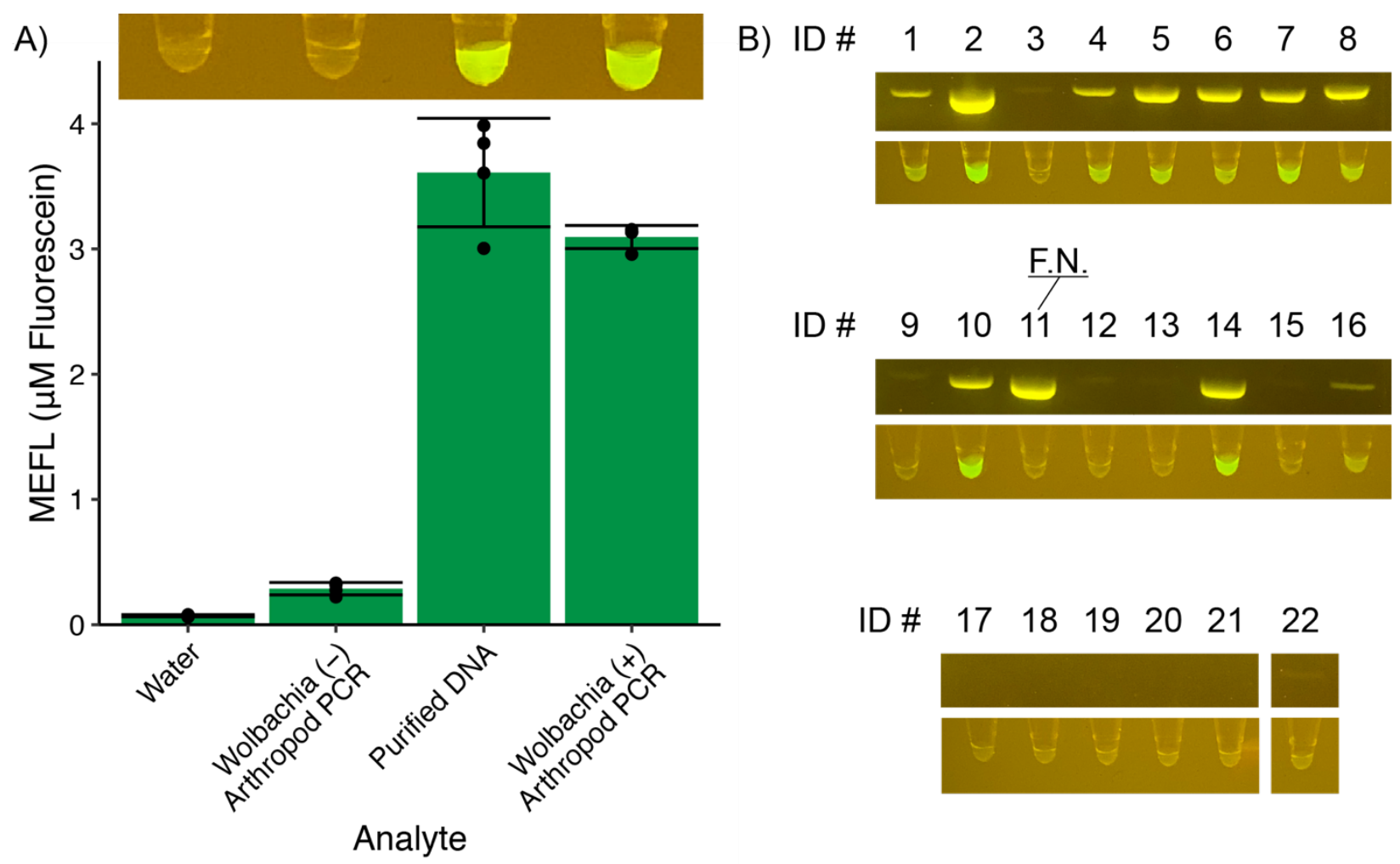

Figure 3. Freeze-dried CRISPR-Cas12 reactions can be used by a high school student to detect Wolbachia infection in field-collected arthropods. A) The inset photo is representative of the control reactions incubated at $37^{\circ} \mathrm{C}$ for 2 hours viewed with a portable blue light imager. B) Arthropods were collected from two locations, Brevard, NC \& Roswell, GA. A high school student extracted DNA from the specimens, amplified Wolbachia DNA using PCR, and concurrently used gel electrophoresis and FD-CC12 reactions to detect the presence of the Wolbachia amplicon. Both the gel and Cas12 reactions were imaged using a portable blue light imager. 
Table 1. Comparison of DNA visualization method equipment and consumable costs. Cost calculation assumes 22 samples and 2 DNA ladders per gel. Comparison does not include the cost of an incubator to incubate rehydrate FD-CC12 reactions as the PCR machine used to generate the PCR product can be used to heat the reaction.

\begin{tabular}{|c|c|c|c|c|c|c|}
\hline Setup & Component & Supplier & $\begin{array}{l}\text { Product } \\
\text { Number }\end{array}$ & Unit Cost & $\begin{array}{c}\text { Initial } \\
\text { Equipment } \\
\text { Investment }\end{array}$ & $\begin{array}{l}\text { Reagent } \\
\text { Cost } / \\
\text { Reaction }\end{array}$ \\
\hline \multirow{7}{*}{$\begin{array}{l}\text { Traditional Gel } \\
\text { Electrophoresis }\end{array}$} & Casting Tray & \multirow{3}{*}{ BioRad } & \multirow{3}{*}{1640300} & \multirow{3}{*}{$\$ 993$} & \multirow{7}{*}{$\$ 993.00$} & \multirow{7}{*}{$\$ 0.31$} \\
\hline & $\begin{array}{c}\text { Electrophoresis } \\
\text { Chamber }\end{array}$ & & & & & \\
\hline & Power Supply & & & & & \\
\hline & Agarose & BioRad & 1613100 & $\begin{array}{c}\$ 0.84 / \\
\text { gel }\end{array}$ & & \\
\hline & TBE Buffer & BioRad & 1610770 & $\begin{array}{c}\$ 0.66 / \\
\text { gel }\end{array}$ & & \\
\hline & FlashBlue Stain & EDVOTEK & 609 & $\begin{array}{c}\$ 2.63 / \\
\text { gel }\end{array}$ & & \\
\hline & DNA Ladder & BioRad & 1708352 & $\begin{array}{c}\$ 2.76 / \\
\text { gel }\end{array}$ & & \\
\hline \multirow{4}{*}{$\begin{array}{c}\text { Education } \\
\text { Optimized Gel } \\
\text { Electrophoresis }\end{array}$} & $\begin{array}{c}\text { blueGel }^{\mathrm{TM}} \\
\text { electrophoresis with } \\
\text { built-in transilluminator }\end{array}$ & MiniPCR & $\begin{array}{l}\text { QP-1500- } \\
01\end{array}$ & $\$ 300.00$ & \multirow{4}{*}{$\$ 300.00$} & \multirow{4}{*}{$\$ 0.15$} \\
\hline & $\begin{array}{c}\text { GelGreen }{ }^{\circledR} \text { Agarose } \\
\text { Tabs }^{\mathrm{TM}}\end{array}$ & MiniPCR & $\begin{array}{l}\text { RG-1500- } \\
10\end{array}$ & $\begin{array}{c}\$ 1.20 / \\
\text { gel }\end{array}$ & & \\
\hline & TBE Buffer & MiniPCR & $\begin{array}{l}\text { RG-1502- } \\
02\end{array}$ & $\begin{array}{l}\$ 0.09 / \\
\text { gel }\end{array}$ & & \\
\hline & DNA Ladder & MiniPCR & $\begin{array}{l}\text { RG-1003- } \\
01\end{array}$ & $\begin{array}{c}\$ 2.00 / \\
\text { gel }\end{array}$ & & \\
\hline \multirow{3}{*}{$\begin{array}{c}10 \mu \mathrm{L} \text { Tube-Based } \\
\text { FD-CC12 }\end{array}$} & $\begin{array}{c}\text { P51 }{ }^{\mathrm{TM}} \text { Molecular } \\
\text { Fluorescence Viewer }\end{array}$ & MiniPCR & $\begin{array}{l}\text { QP-1900- } \\
01\end{array}$ & $\$ 28.00$ & \multirow{3}{*}{$\$ 28.00$} & \multirow{3}{*}{$\$ 0.47$} \\
\hline & Freeze-Dried Reaction & $\begin{array}{l}\text { See Table } \\
\text { S3 }\end{array}$ & $\begin{array}{l}\text { See Table } \\
\text { S3 }\end{array}$ & $\begin{array}{l}\$ 0.2907 / \\
\text { reaction }\end{array}$ & & \\
\hline & PCR Tube & $\begin{array}{l}\text { Thermo } \\
\text { Scientific }\end{array}$ & AB2000 & $\begin{array}{l}\$ 0.18 / \\
\text { reaction }\end{array}$ & & \\
\hline \multirow{3}{*}{$\begin{array}{c}1.8 \mu \mathrm{L} \text { Paper-Based } \\
\text { FD-CC12 }\end{array}$} & $\begin{array}{c}\text { P51 }{ }^{\mathrm{TM}} \text { Molecular } \\
\text { Fluorescence Viewer }\end{array}$ & MiniPCR & $\begin{array}{l}\text { QP-1900- } \\
01\end{array}$ & $\$ 28.00$ & \multirow{3}{*}{$\$ 28.00$} & \multirow{3}{*}{$\$ 0.05$} \\
\hline & Freeze-Dried Reaction & $\begin{array}{l}\text { See Table } \\
\text { S3 }\end{array}$ & $\begin{array}{l}\text { See Table } \\
\text { S3 }\end{array}$ & $\begin{array}{l}\$ 0.0523 / \\
\text { reaction }\end{array}$ & & \\
\hline & Filter Paper & $\begin{array}{l}\text { Cole- } \\
\text { Parmer }\end{array}$ & $\begin{array}{c}\text { UX- } \\
06647-20\end{array}$ & $\begin{array}{l}\$ 0.0007 \text { / } \\
\text { reaction }\end{array}$ & & \\
\hline
\end{tabular}

